\title{
TREAT-TO-TARGET IN OSTEOPOROSIS. MYTH OR REALITY? RESULTS OF A SPANISH DELPHI STUDY
}

M. Muñoz-Torres ${ }^{1}$, E. Casado ${ }^{2}$, E. Jódar ${ }^{3}$, X. Nogués ${ }^{4}$, JM. Nolla ${ }^{5}$, JM. Quesada-Gómez ${ }^{6}$, L. Canals 7 , M. Balcells 7, L. Lizán 8

1. University Hospital San Cecilio, Granada, Spain; 2. University Hospital Parc Tauli, Sabadell, Spain; 3. University Hospital Quirón, Madrid, Spain; 4. Mar Institute of Medical Research (IMIM), Barcelona ; 5. University Hospital Bellvitge, L'Hospitalet de Llobregat, Spain; 6. University Hospital Reina Sofía. IMIBIC. RETICEF, Córdoba, Spain; 7. Amgen, Barcelona, Spain; 8. Outcomes'10, University Jaume I, Castelló de la Plana, Spain

\section{Background}

Treat-to-Target (T2T) strategy, that uses well defined and specific targets to control a disease, has been widely used in chronic conditions that have well-established treatment goals such as rheumatoid arthritis (1) and diabetes mellitus (2)

Some authors have proposed that a T2T strategy could be useful in osteoporosis (3-5). However, in the treatment of osteoporosis there are no established consensus goals, hindering the implementation of this strategy.

The Delphi technique is a structured method widely used to gather and obtain important information regarding a specific topic. Its key features are anonymity between participants and a controlled feedback (6).

Objective

To define, by expert consensus, the criteria for the application of a T2T strategy in osteoporosis, in Spain, and to assess the adequacy of current treatments for it.

Material and methods

Scientific Committee

Six Spanish experts in osteoporosis ( 3 endocrinologists, 2 rheumatologists and 1 internist) formed the Scientific Committee that led the project.

Study design

The Scientific Committee, based on a systematic review of the literature, designed the questionnaire used in two Delphi rounds.

A total of 24 items were included in the questionnaire in order to assess the experts' wish (W) and prognosis $(P)$ for each item to occur in 5-year time.

The Scientific Committee with the collaboration of the Spanish Society of Rheumatology (SER) and the Spanish Society for Bone and Mineral Metabolism (SEIOMM) selected the panel of experts to participate in the two-round Delphi, based on their experience.

Two rounds of Delphi were completed.

Experts valued each item in a 7-point Likert scale (1=entirely disagree; 7=entirely agree).

Consensus was defined as $\geq 75 \%$ of agreement (5-7) or disagreement (1-3) responses.

- It was defined that the agreement or disagreement consensus was strong when more than $75 \%$ of the participants responded entirely agree / mostly agree (6-7) or entirely disagree / mostly disagree (1-2). The second round questionnaire only included those items without consensus in the first round.

Data Analysis

A database using the SPSS v.20 was created.

Descriptive statistics were applied to determine consensus and quantify its degree. Percentage of panelists with the same response to the same statement was calculated for the 1st and the 2nd rounds.

Results

Characteristics of Delphi panel

The $1^{\text {st }}$ round was completed by 112 out of 166 experts $(67.5 \%)$ and $106(94.6 \%)$ completed the $2^{\text {nd }}$. Most of the participants were rheumatologists (59.8\%); followed by internists (14.3\%) and endocrinologists (10.7\%)

Participants had a mean clinical experience of 21.3 years (SD: 8.5)

After the two-round Delphi, there was consensus on $70 \%$ of items. In $44 \%$ of them the consensus was achieved in the $1^{\text {st }}$ round Delphi.

Applicability of the Treat-to-Target strategy in osteoporosis

Figure 1. Applicability of the Treat-to-Target strategy in osteoporosis
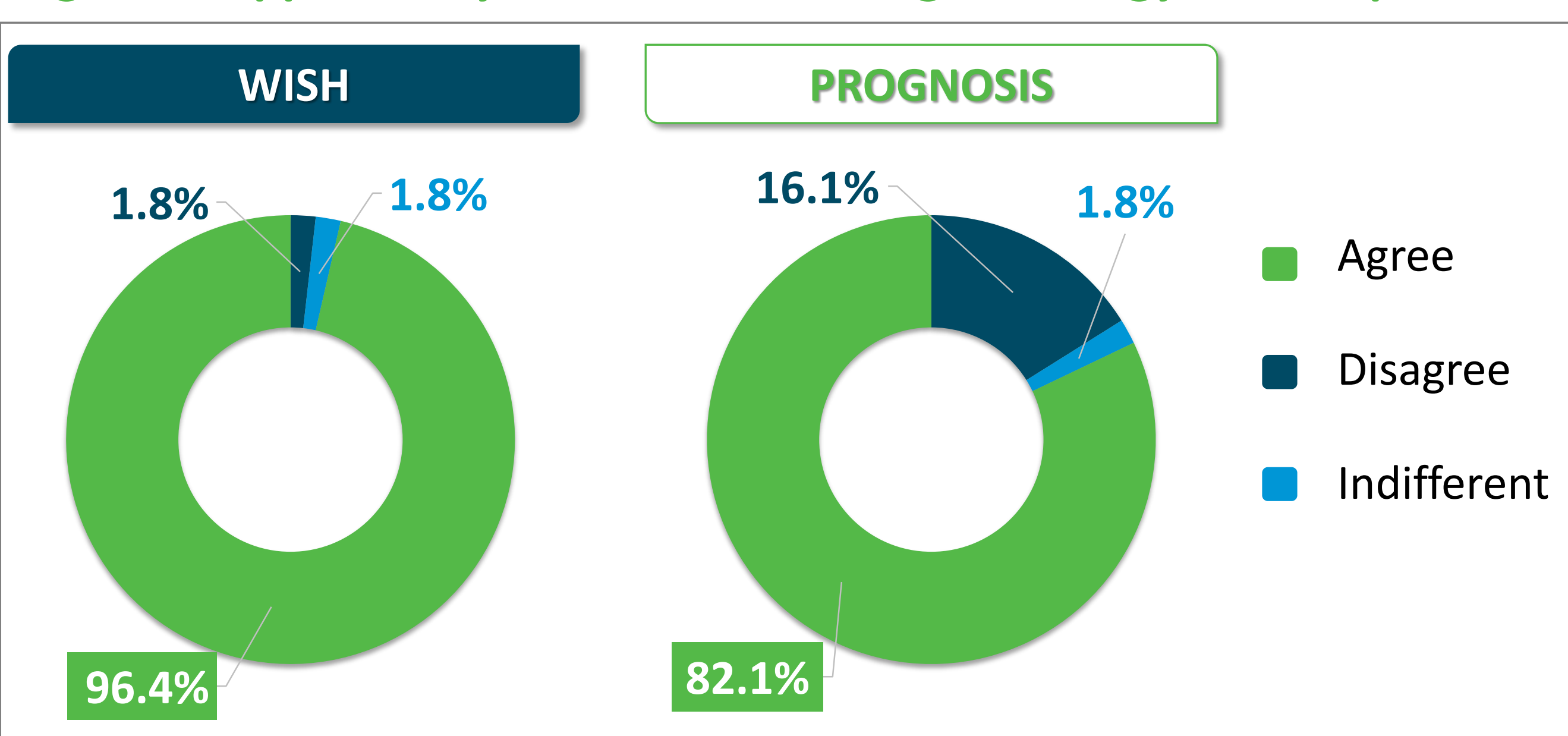

In the $1^{\text {st }}$ round Delphi there was consensus on an implementation of a T2T strategy in the management of patients with osteoporosis, although this consensus was higher in wish $(96 \%)$ than in prognosis (82\%) (Figure 1).

Therapeutic objectives to be established in T2T strategy in osteoporosis

Experts agreed on the utility of improvement Bone Mineral Density (BMD) (W: 91.1\%; P: 91.1\%), fracture risk reduction by FRAX (W: 75.9\%; P: $84.0 \%$ ) and lack of fractures (W: $99.1 \%$; P: $97.3 \%$ ) as therapeutic objectives (Figure 2).

Figure 2. Therapeutic objectives to be established in T2T strategy in osteoporosis (wish and prognosis).
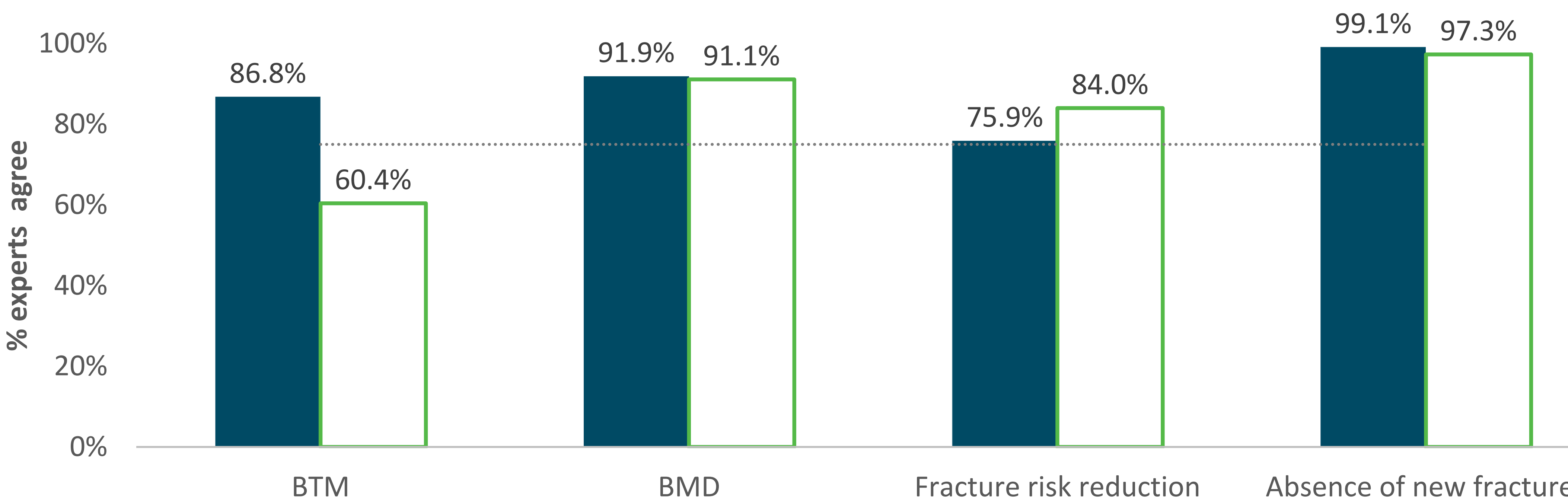

BTM

BMD

Fracture risk reduction
(FRAX)

Absence of new fractures

WISH DPROGNOSIS
Treatment failure definition

Treatment failure was defined as no BMD gain after 2 (W:81.3\%; P:82.1) or 3 years (W:77.7\%; P:75.9\%) (Figure 3), new fracture diagnosis within 2 (W:92.0\%; P:92.0) or 3 years (W:90.2\%; P:88.4\%) (Figure 4) or the absence of Bone Turnover Markers (BTM) change after 6 months (W:75.0\%; P:93.4\%) or 1 year (W:90.6\%; P:89.6\%) of treatment (Figure 5).

Figure 3. Treatment failure defined using BMD gain.
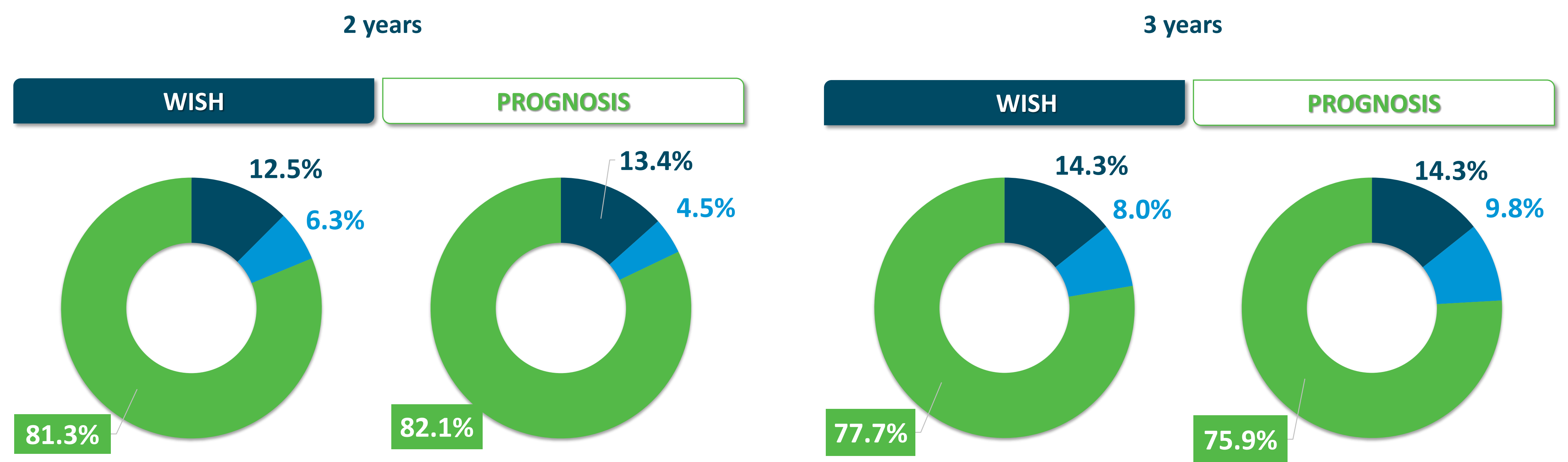

Agree Disagree Indifferent

Figure 4. Treatment failure defined using new fracture diagnosis

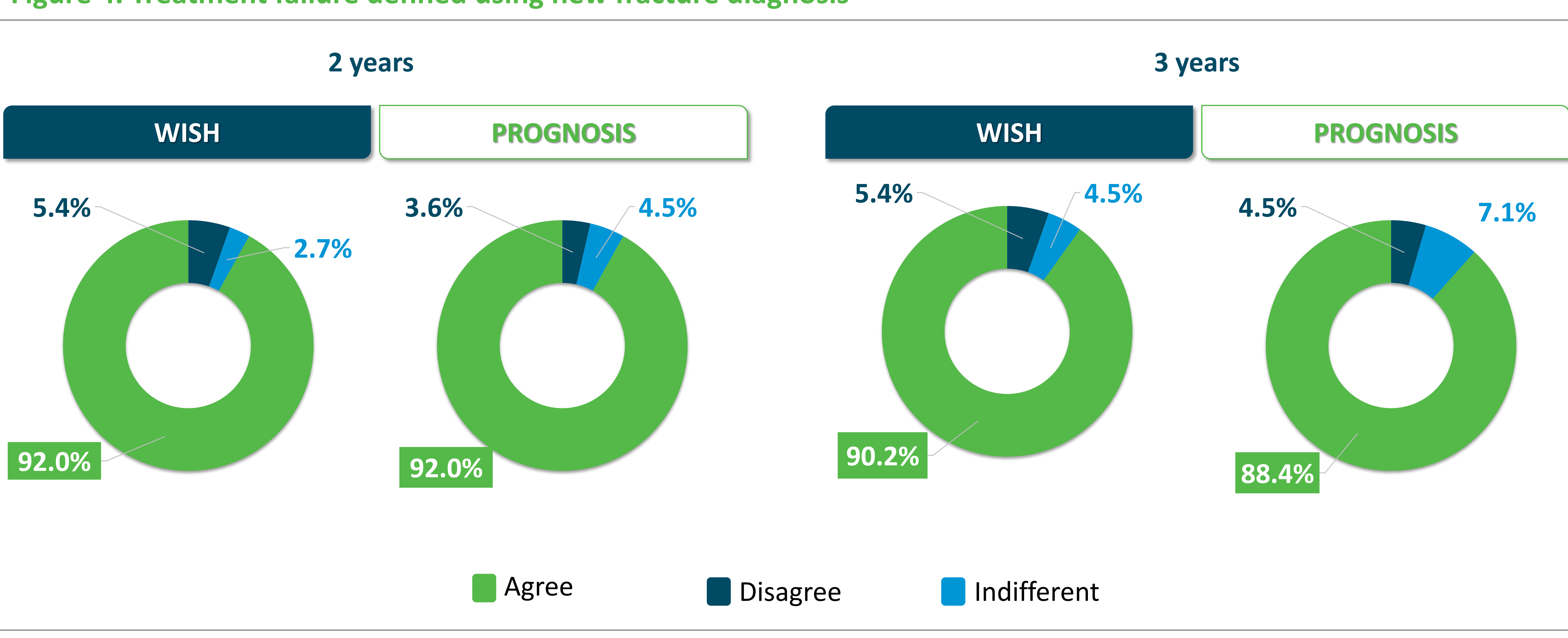

Figure 5. Treatment failure defined using absence of BTM change

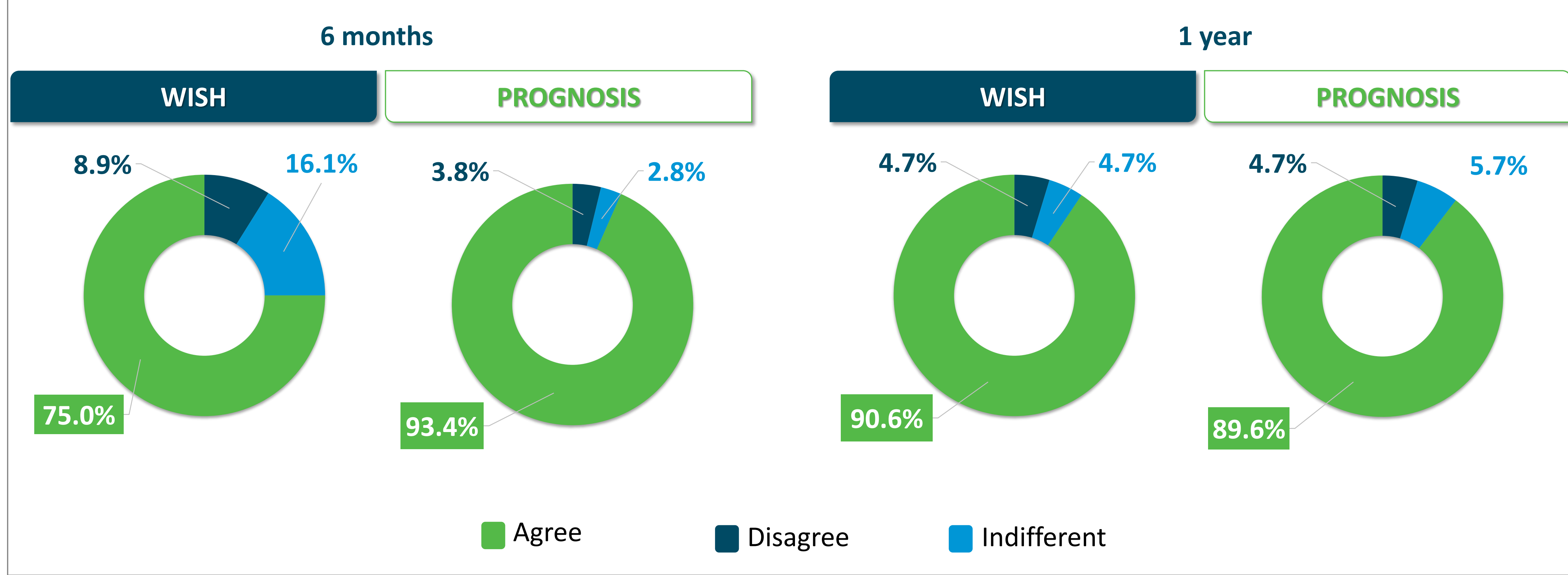

Treatments to be prescribed in the Treat-to-target strategy on osteoporosis.

Except for strontium ranelate (W:76.4\%; P:58.5\%), consensus was reached for all available and upcoming novel therapies to achieve a therapeutic target through T2T strategy application (Figure 6).

Figure 6. Treatments to be prescribed in the Treat-to-target strategy on osteoporosis

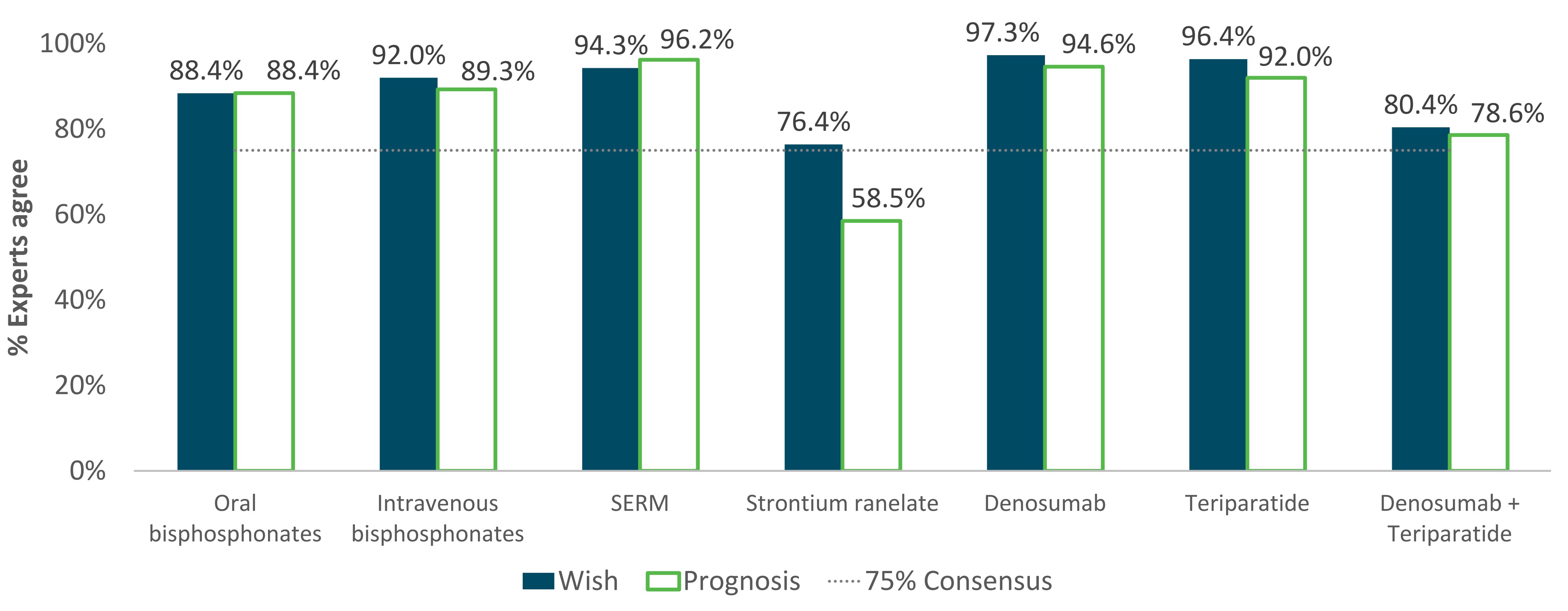

Conclusion

A T2T strategy in osteoporosis can be implemented in Spain, since therapeutic objectives, treatment failure definition and appropriate treatment choice for this strategy have been established.

Disclosures

Amgen S.A. sponsored this study and provided assistance with the preparation of this poster. MMT research grants from Amgen, Lilly, consultant for Amgen, Lilly, Alexion; speakers' bureau for Amgen, Lilly. EC speakers' bureau for Amgen, Lilly. EJT research grants from Amgen, MSD, consultant for Amgen, Lilly, speakers' bureau for Amgen, AALS, lantarmaco, Lily. XN research grants from Lily, Amgen

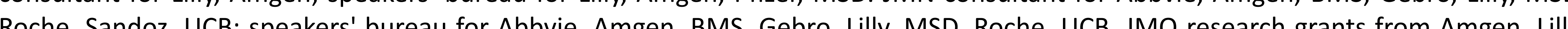
FIS Carlos III, RETICEF, Intensification of Investigation Junta de Andalucía: consultant for Amgen, MSD, Italfarmaco, FAES; speakers' bureau for Amgen, MSD, Italfarmaco, FAES. LC and MB are employees of Amgen and may have Amgen stock/stock options. $L$ has no conflicts of interest to disclose.

References

. Smolen, et al. Ann Rheum Dis. 2014;73(3):492-509.3; 2. Inzucchi, et al. Diabetol. 2015;58:429-442; 3. Lewiecki et al. J Clin Endocrino Metab 2013; 98: 946-53; 4. Cummings et al. J Bone Miner Res 2013; 28:433-438; 5. Chapurlat. Joint Bone Spine 2016 Feb 10. [Epub ahead of print;; 6 . Dia 